\title{
A Double Tiling of Triangles and Regular Hexagons
}

\author{
H. Okumura ${ }^{1}$ and J. F. Rigby ${ }^{2}$ \\ ${ }^{1}$ Maebashi Institute of Technology, \\ 460 Kamisadori, Maebashi, Gunma 371, Japan \\ okumura@maebashi-it.ac.jp \\ ${ }^{2}$ School of Mathematics, Cardiff University, \\ Senghennydd Road, Cardiff CF2 4YH, Wales \\ rigby@cardiff.ac.uk
}

\begin{abstract}
A tiling of triangles and regular hexagons, which wraps around a focal point and covers the plane twice, is investigated using both synthetic triangle geometry and complex numbers.
\end{abstract}

\section{Introduction}

In attempting to generalize a theorem of traditional Japanese geometry involving squares [1, p. 47], the first author proved the existence of an interesting figure consisting of a regular hexagon surrounded by six others, as shown in Fig. 1. He then noticed that this figure could be used to construct a tiling of the plane by triangles and regular hexagons as in Fig. 4. On the basis of a computer drawing he conjectured that although the tiling fails to fit together in a simple manner (Fig. 4), it always joins up with itself after wrapping twice around an origin or focal point (Fig. 5) to produce a "double tiling"; this is an unusual and interesting situation. He further conjectured that the vertices of the tiling and the centres of the hexagons lie on various families of parabolas with their foci at the focal point of the tiling. In this article we give a simpler proof that the situation shown in Fig. 1 always exists if we start off with three regular hexagons erected on the sides of any triangle. We then verify the conjectures about the tiling, except that the foci of the parabolas do not all coincide at the focal point of the tiling.

Our initial investigation of these double tilings in Sections 1 and 2 demonstrates an interesting use of triangle geometry (in particular, properties of the Fermat points and isodynamic points of a triangle). We use a completely different method to finish the investigation: in Section 4 we show that the double tilings arise when we apply an unusual type of transformation of points in the complex plane to the quasiregular tiling $(3,6,3,6)$, but we still need to use an important result from Section 2 to show that all double tilings arise in this way. 


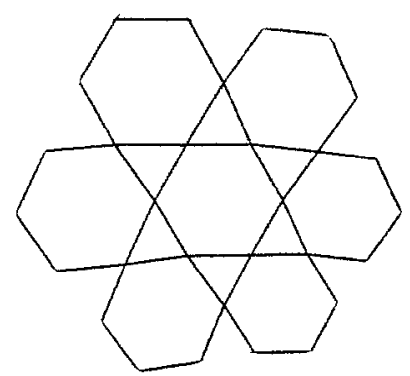

Fig. 1

\section{A Regular Hexagon Surrounded by Six Others}

Theorem 1.1. Let $A_{1} A_{2} A_{3} A_{4} A_{5} A_{6}$ be a regular hexagon erected on the side $A_{2} A_{1}$ of any triangle $A_{2} A_{1} B_{2}$ as shown in Fig. 2. Then there exists a ring of six regular hexagons $B_{n+1} A_{n} B_{n} \cdots(n=1,2, \ldots, 6)$ surrounding the hexagon $A_{1} A_{2} A_{3} A_{4} A_{5} A_{6}$ as shown in the figure, where suffixes are calculated modulo 6.

Proof. Let $C$ be the reflection of $B_{2}$ in $A_{1} A_{2}$, and let $B_{n+1}$ be the reflection of $C$ in $A_{n} A_{n+1}(n=1,2, \ldots, 6)$. Then $A_{1} B_{2}=A_{1} C=A_{1} B_{1}$ because reflections preserve

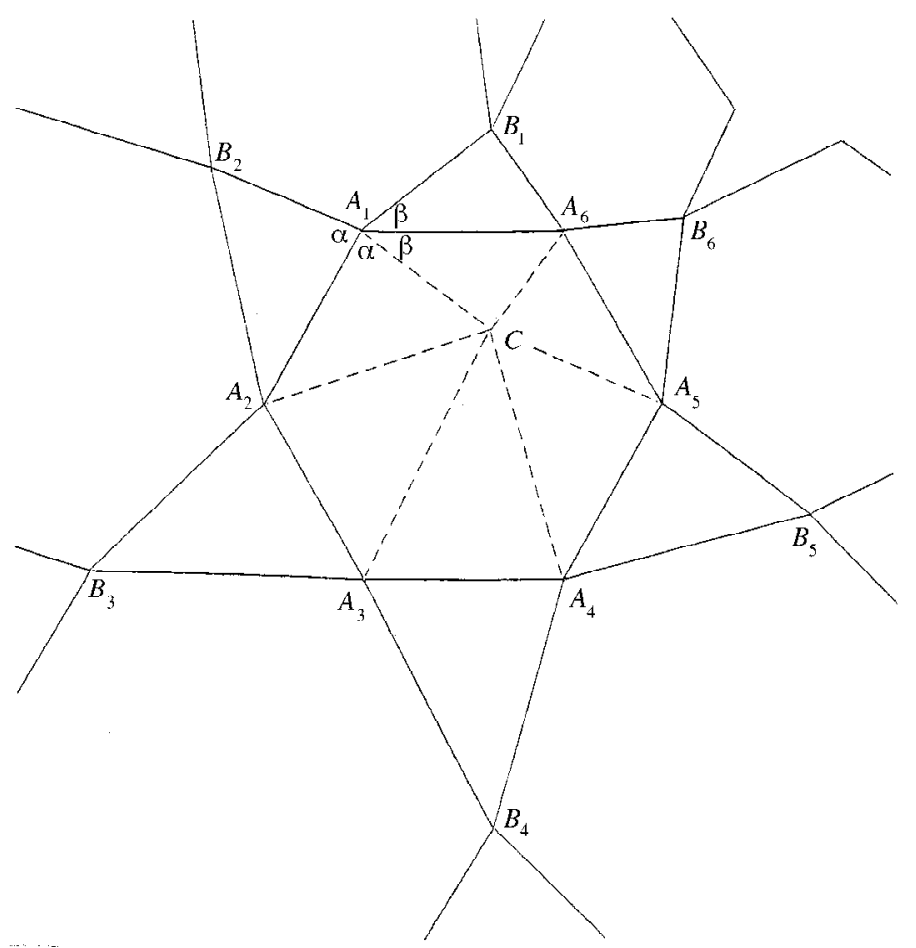

Fig. 2 


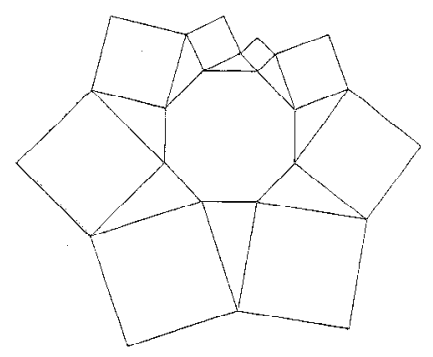

Fig. 3

lengths. Also the two angles marked $\alpha$ are equal, as are those marked $\beta$. However, $120^{\circ}=\angle A_{2} A_{1} A_{6}=\alpha+\beta$. Hence $\angle B_{1} A_{1} B_{2}=120^{\circ}$. Hence $B_{2} A_{1}$ and $A_{1} B_{1}$ are consecutive vertices of a regular hexagon $B_{2} A_{1} B_{1} \cdots$. Similarly for the other hexagons in the figure.

Remark. This proof can be adapted to show the existence of twelve equilateral triangles surrounding a regular dodecagon, eight squares surrounding a regular octagon (Fig. 3), and five regular decagons surrounding a regular pentagon; in the last case at least two of the decagons will always overlap (except when $C$ lies at the centre of the pentagon, in which case each decagon touches its neighbours along an edge). These are all special cases of a general result: if $2 / n+1 / k=\frac{1}{2}$ we can obtain a regular $n$-gon surrounded by $n$ regular $k$-gons; $n$ and $k$ need not be integers, and by taking $n=4$ and $k=\infty$ we can even include the apeirogon (a regular polygon with $180^{\circ}$ angles).

Note that all the hexagons discussed are regular, unless otherwise stated, so we use the word "hexagon" to mean "regular hexagon." We regard each hexagon as being bounded by six directed line segments, and as being positively oriented (abbreviated to "positive"), so that as we travel around the hexagon in the direction of the directed segments the hexagon is on our left. Triangles occurring in a tiling or in part of a tiling (as in Figs. 2 and 4 for example) will be labelled and oriented in such a way that a triangle and a hexagon sharing an edge induce opposite orientations on that edge. It turns out that most of the triangles occurring in a tiling will be positive as in Fig. 4, but Theorem 1 remains true if $A_{2} A_{1} B_{2}$ (or any other triangle in Fig. 2) is negative or even if $A_{2}, A_{1}$ and $B_{2}$ are collinear. If in Fig. 2 the angle $A_{1} B_{1} A_{6}$ (for example) were greater than $120^{\circ}$, then the hexagons $B_{2} A_{1} B_{1} \cdots$ and $B_{1} A_{6} B_{6} \cdots$ would overlap.

Each of the six new hexagons in Theorem 1.1 is already partially surrounded by three hexagons, and we can use the theorem to obtain a ring of six regular hexagons surrounding each new hexagon. Proceeding in this way we should presumably be able to produce a tiling of alternate triangles and regular hexagons covering the entire plane. However, a computer drawing of a typical situation (Fig. 4) shows two interesting facts: (a) the hexagons appear to get smaller as we move towards some kind of focal point of the tiling, and (b) if we continue the tiling around this focal point it fails to "join up" with itself. However, if we continue the tiling around the focal point in a second layer, it 


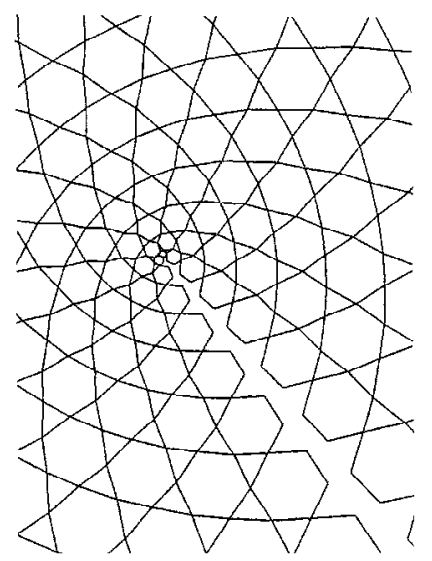

Fig. 4

apparently joins up after we have gone around the focal point twice, as in Fig. 5. Does this always happen?

We refer to a tiling of triangles and regular hexagons constructed in the above manner as an $h$-tiling. We show that each h-tiling does have a definite focal point, and that the vertices of the tiling always lie on a two-sheeted surface whose branch point lies at the focal point. There is a stronger definition of the term "double tiling" which is discussed in Section 4, and we only conjecture that h-tilings satisfy this stronger condition; but we can point out now that near the focal point of an h-tiling, negative triangles will occur, as in Fig. 6 which shows a typical situation, and we must regard such triangles as providing a negative contribution to the tiling. For instance, the immediate vicinity of the focal point in Fig. 6 (indicated by a dot) is contained in three hexagons and one negative triangle, and there are points near the focal point contained in four hexagons and two negative triangles.

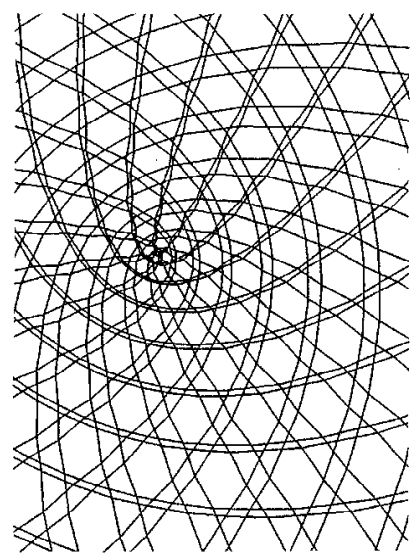

Fig. 5 


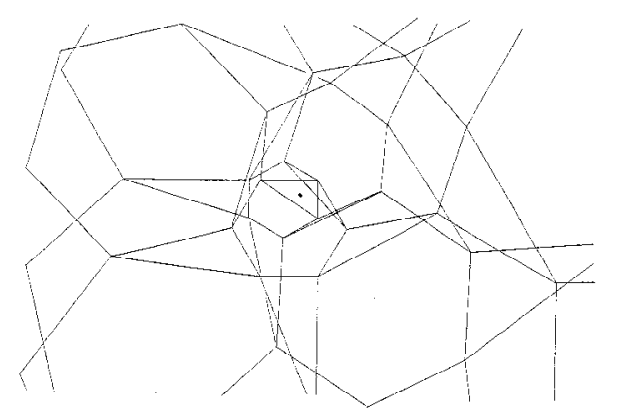

Fig. 6

There are two special cases, shown in Figs. 7 and 8, when the patterns of tiles on the two sheets coincide to produce a tiling that can be described as a simple tiling except near the focal point; we show at the end of Section 4 that these are the only such special cases. (The well-known quasiregular tiling $(3,6,3,6)$ of equilateral triangles and regular hexagons covers the plane only once; it does not have a focal point, and its vertices lie on lines rather than parabolas, so we do not regard it as a special case of an h-tiling.)

In Fig. 2 the point $C$ can be called the tiling centre, or t-centre, of the hexagon $A_{1} A_{2} A_{3} A_{4} A_{5} A_{6}$. Every hexagon of an h-tiling has a t-centre, as well as what we may call a true centre. Since we are excluding from our discussion the quasiregular tiling $(3,6,3,6)$ (at least until we use it in Section 4), the t-centre of each hexagon is distinct from its true centre. The line joining the $\mathrm{t}$-centre to the true centre is called the $t$-line of the hexagon. A practical investigation of Fig. 4 led to the conjecture that the t-lines of the hexagons of an h-tiling all pass through a common point; this is the point that we define as the focal point of the tiling. The search for a proof of this conjecture using synthetic methods leads to an interesting theorem in triangle geometry, as described in the next section.

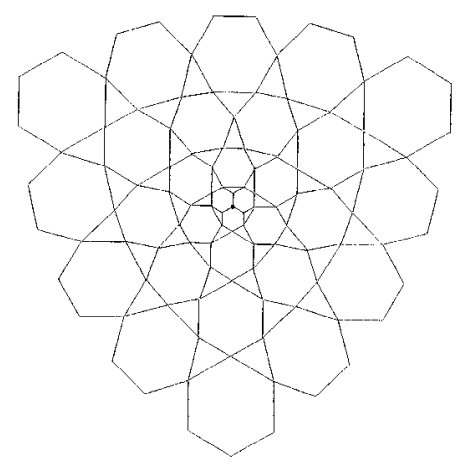

Fig. 7 


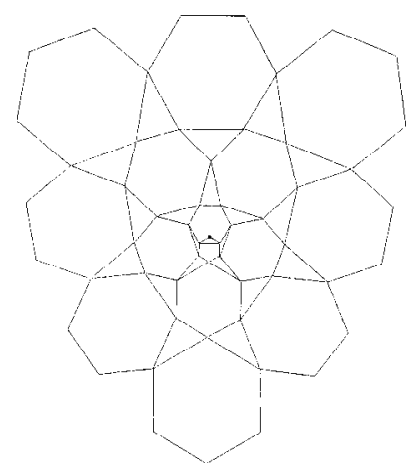

Fig. 8

\section{Fermat Points and Isodynamic Points}

Results in this section that are stated without proof can be found in various standard texts, such as [2]. A very useful list of special points connected with a triangle, including the Fermat points and isodynamic points, can be found in [3]. In the proofs we use directed angles between lines, determined modulo $180^{\circ}$, positive angles being measured anticlockwise; $\angle A B C$ will now denote the directed angle measured from the line $B A$ to the line $B C$.

We construct positive equilateral triangles $P_{1} C B, Q_{1} A C, R_{1} B A$ on the sides of a triangle $A B C$, so that $P_{1}$ is on the right of the directed segment $B C$, etc. Then $P_{1} Q_{1} R_{1}$ is called the right Fermat triangle of $A B C$ (see Fig. 9). The lengths $A P_{1}, B Q_{1}, C R_{1}$ are equal, the lines $A P_{1}, B Q_{1}, C R_{1}$ meet at the right Fermat point $F_{1}$ of $A B C$ (not shown in the figure) and $\angle B F_{1} C=\angle C F_{1} A=\angle A F_{1} B=-60^{\circ}$. Similarly if negative equilateral triangles $P_{2} C B, Q_{2} A C, R_{2} B A$ are constructed on the sides of $A B C$, then $P_{2} Q_{2} R_{2}$ is the left Fermat triangle of $A B C$, the lengths $A P_{2}, B Q_{2}, C R_{2}$ are equal, the lines $A P_{2}$, $B Q_{2}, C R_{2}$ meet at the left Fermat point $F_{2}$, and $\angle B F_{2} C=\angle C F_{2} A=\angle A F_{2} B=60^{\circ}$. (When $A B C$ is a positive triangle as in Fig. 9, the equilateral triangles $P_{1} C B$, etc. are constructed outwards on the sides of $A B C ; P_{1} Q_{1} R_{1}$ is then usually called the first Fermat triangle and $F_{1}$ the first Fermat point. However, when $A B C$ is a negative triangle, $P_{1} C B$, etc. are constructed inwards, so $P_{1} Q_{1} R_{1}$ and $F_{1}$ become the second Fermat triangle and point. Our present terminology will be more useful here: the definitions of right and left Fermat points remain valid even when $A, B$ and $C$ are collinear, when the distinction between first and second Fermat points no longer makes sense.)

Denote the sides $B C, C A, A B$ of triangle $A B C$ by $a, b, c$, and denote the lines $A P_{2} F_{2}, B Q_{2} F_{2}, C R_{2} F_{2}$ by $p, q, r$ (see Fig. 9). The line $p^{*}$ such that $\angle b p^{*}=\angle p c$ (or equivalently $\left.\angle c p^{*}=\angle p b\right)$ is called the isogonal line of $p$ with respect to the triangle $A B C$. The isogonal lines $q^{*}$ of $q$ and $r^{*}$ of $r$ are defined similarly, so that

$$
\left.\angle c q^{*}=\angle q a \quad \text { (or equivalently } \angle q^{*} a=\angle c q\right) \quad \text { and } \quad \angle a r^{*}=\angle r b \text {, }
$$

as shown in the figure. Since $p, q, r$ are concurrent at $F_{2}$, it follows from the isogonal conjugate theorem that the lines $p^{*}, q^{*}, r^{*}$ are concurrent at a point $\Omega_{2}$, the isogonal conjugate of $F_{2} ; \Omega_{2}$ is called the left isodynamic point of $A B C$. 


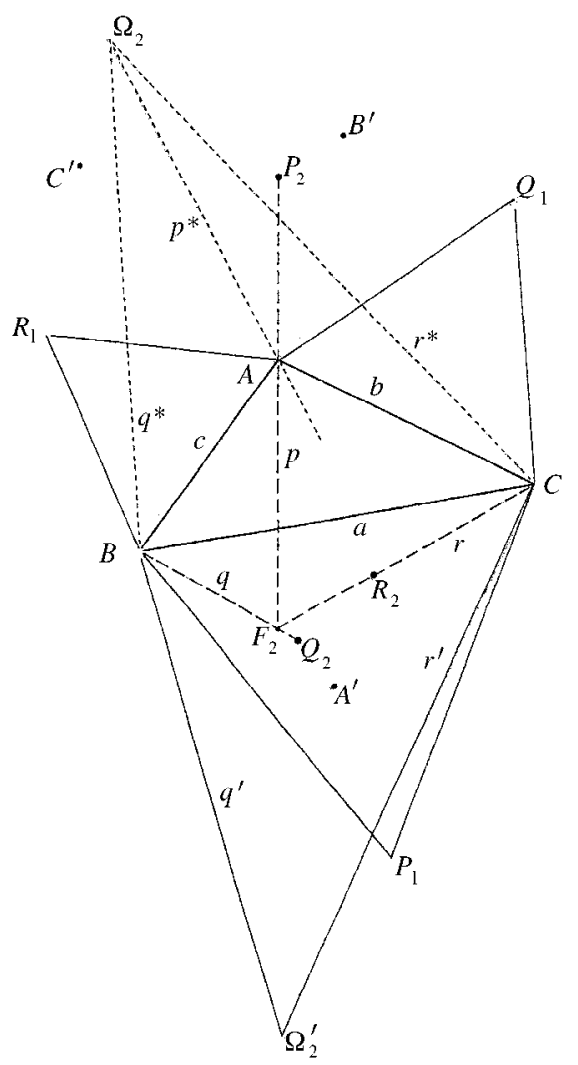

Fig. 9

The reflections $A^{\prime}, B^{\prime}, C^{\prime}$ of $A, B, C$ in $B C, C A, A B$ respectively form the triangle of the three images.

Theorem 2.1. $P_{1} A^{\prime}, Q_{1} B^{\prime}, R_{1} C^{\prime}$ meet at $\Omega_{2}$, and the lengths $P_{1} A^{\prime}, Q_{1} B^{\prime}, R_{1} C^{\prime}$ are equal.

Proof. Refer again to Fig. 9. Denote the reflections of $q^{*}$ and $r^{*}$ in $a$ by $q^{\prime}$ and $r^{\prime}$; so that $q^{\prime}$ and $r^{\prime}$ meet at $\Omega_{2}^{\prime}$, the reflections of $\Omega_{2}$ in $a$. Then $\angle a q^{\prime}=\angle q^{*} a=\angle c q$ and $\angle a r^{\prime}=\angle r^{*} a=\angle b r$ (using (1)); also $\angle q p=\angle p r=-60^{\circ}$. Hence, $p, q^{\prime}, r^{\prime}$ are the isogonal lines of $p, c, b$ with respect to the triangle $F_{2} B C$; but $p, c, b$ are concurrent at $A$, and hence by the isogonal conjugate theorem $p, q^{\prime}, r^{\prime}$ are concurrent, at $\Omega_{2}^{\prime}$; so $P_{2}, A, F_{2}, \Omega_{2}^{\prime}$ are collinear.

Reflecting in $a$ we deduce that $P_{1}, A^{\prime}, \Omega_{2}$ are collinear, i.e. $P_{1} A^{\prime}$ passes through $\Omega_{2}$. Similarly $Q_{1} B^{\prime}$ and $R_{1} C^{\prime}$ pass through $\Omega_{2}$. Also $P_{1} A^{\prime}=P_{2} A$, etc., and we know that $P_{2} A=Q_{2} B=R_{2} C$. Hence $P_{1} A^{\prime}=Q_{1} B^{\prime}=R_{1} C^{\prime}$ and the proof is complete.

We can of course prove similarly that $P_{2} A^{\prime}, Q_{2} B^{\prime}, R_{2} C^{\prime}$ meet at $\Omega_{1}$, the isogonal conjugate of $F_{1}$ with respect to $A B C$, and that $P_{2} A^{\prime}=Q_{2} B^{\prime}=R_{2} C^{\prime} ; \Omega_{1}$ is called the right isodynamic point of $A B C$. 


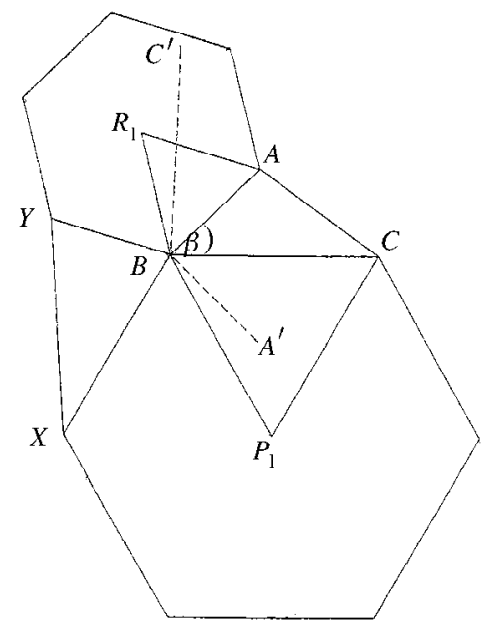

Fig. 10

Now we consider $A B C$ in Fig. 9 to be a typical triangle of an h-tiling. Then $P_{1}, Q_{1}, R_{1}$ are the true centres of the surrounding hexagons, and $A^{\prime}, B^{\prime}, C^{\prime}$ are their t-centres. Thus Theorem 2.1 states that the t-lines of these three hexagons are concurrent, at the left isodynamic point of the triangle $A B C$.

Theorem 2.2. Two adjacent triangles of an h-tiling (i.e. two triangles with a common vertex) have the same left isodynamic point.

Proof. Consider two triangles of an h-tiling, $A B C$ and $X B Y$, with common vertex $B$, as in Fig. 10 where the same notation as in Fig. 9 is used. The lines $P_{1} A^{\prime}$ and $R_{1} C^{\prime}$ are the t-lines of the hexagons $C B X \cdots$ and $Y B A \cdots$; if these t-lines are distinct, they meet at the left isodynamic point of $A B C$ by Theorem 2.1 , and also at the left isodynamic point of $X B Y$ by the same theorem; the result is therefore true when the t-lines are distinct. Now if we rotate triangle $B A^{\prime} P_{1}$ about $B$ through an angle $2 \beta+60^{\circ}$, where $\beta$ is the angle $C B A$, we get triangle $B R_{1} C^{\prime}$. Hence the lines $A^{\prime} P_{1}$ and $R_{1} C^{\prime}$ are not parallel and are therefore distinct, unless $\beta=60^{\circ}$ or $150^{\circ}$ (we need only consider $\beta$ modulo $180^{\circ}$ ). When $\beta=60^{\circ}$ we obtain a symmetrical figure of two hexagons and two triangles, with $P_{1} R_{1}$ as the line of symmetry, but the other case is more difficult to deal with directly; so the simplest procedure seems to be to consider two hexagons of fixed sizes, one of which is allowed to rotate about $B$ : the result is true except in the special cases, and hence by continuity it is true in the special cases also, because the left isodynamic points exist even in the special cases.

Corollary 2.3. All the triangles of an h-tiling have the same left isodynamic point, and the t-lines of all the hexagons of the tiling pass through this point.

This point is called the focal point of the tiling. 


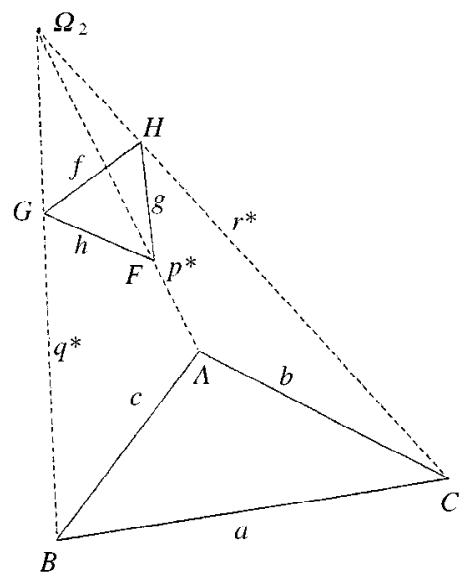

Fig. 11

Corollary 2.4. The distance between the t-centre and the true centre is the same for all hexagons in an h-tiling (this follows from Theorems 2.1 and 2.2).

One of the most important properties of isodynamic points, which will be required in Section 4, is given in the next theorem. It is a standard result, except perhaps for the precise statements involving orientation, but the proof given in [2] can be simplified.

Theorem 2.5. The inverses of the vertices of a triangle, with respect to a circle whose centre is at the left isodynamic point, form a negative equilateral triangle.

Proof. In Fig. 11, which must be combined with Fig. 9, F, G, $H$ are the inverses of $A, B, C$ with respect to a circle with centre $\Omega_{2}$ and radius $k$. Denote the sides $G H, H F, F G$ of triangle $F G H$ by $f, g, h$, respectively. Since by the definition of inversion $\Omega_{2} F \cdot \Omega_{2} A=\Omega_{2} G \cdot \Omega_{2} B=k^{2}$, the triangles $\Omega_{2} F G$ and $\Omega_{2} B A$ are oppositely similar since they share a common angle at $\Omega_{2}$. Hence $\angle p^{*} h=\angle c q^{*}$; similarly $\angle g p^{*}=\angle r^{*} b$. Hence $\angle g h=\angle g p^{*}+\angle p^{*} h=\angle r^{*} b+\angle c q^{*}=\angle a r+\angle q a=\angle q r$. If we rotate triangle $A B Q_{2}$ about $A$ through an angle $60^{\circ}$ we obtain triangle $A R_{2} C$; hence $\angle q r=60^{\circ}$. Hence $\angle g h=\angle q r=60^{\circ}$. Similarly $\angle h g=\angle f g=60^{\circ}$. Hence $F G H$ is a negative equilateral triangle.

\section{Interlude: An $18_{3}$ Configuration}

Interesting geometrical situations often occur unexpectedly; in this brief interlude we consider a configuration that arises from Theorem 2.1 but is not relevant to the rest of the article. The 16 important points referred to in the previous section are collinear in sets of three as follows: $A P_{1} F_{1}, B Q_{1} F_{1}, C R_{1} F_{1} ; A P_{2} F_{2}, B Q_{2} F_{2}, C R_{2} F_{2} ; A^{\prime} P_{1} \Omega_{2}$, $B^{\prime} Q_{1} \Omega_{2}, C^{\prime} R_{1} \Omega_{2} ; A^{\prime} P_{2} \Omega_{1}, B^{\prime} Q_{2} \Omega_{1}, C^{\prime} R_{2} \Omega_{1}$. If we introduce two more points, the 
orthocentre $H$ and the circumcentre $O$ of triangle $A B C$, we obtain six more collinearities: $A A^{\prime} H, B B^{\prime} H, C C^{\prime} H, P_{1} P_{2} O, Q_{1} Q_{2} O, R_{1} R_{1} O$. We now have an $18_{3}$ configuration of 18 points and 18 lines, with each point lying on three lines and each line passing through three points.

This configuration is transitive on lines, but not on points. It can be regarded as three complete quadrangles: $A A^{\prime} P_{1} P_{2}, B B^{\prime} Q_{1} Q_{1}, C C^{\prime} R_{1} R_{1}$, with the six sides of each quadrangle passing through the points $H, O, F_{1}, \Omega_{1}, F_{2}$ and $\Omega_{2}$.

\section{A Transformation in the Complex Plane}

We now construct h-tilings in an entirely different manner, using a transformation of the complex plane that acts on the quasiregular tiling $(3,6,3,6)$. We then show that all h-tilings can be obtained in this way. An account of the use of complex numbers in geometry can be found in standard texts such as [4].

The mapping $\sigma$ defined by $\sigma(z)=z^{2}$ is a continuous one-to-one mapping of the complex plane onto a two-sheeted surface with a branch point at the origin. This mapping $\sigma$ is a conformal mapping: it preserves angles. Also $\sigma$ maps any line not through the origin to a parabola with focus at the origin; this may be shown as follows. The line $y=a(a \neq 0)$ makes an angle zero with the initial line in the complex plane. A typical point on this line is $p+i a$; this point is transformed by $\sigma$ to $\left(p^{2}-a^{2}\right)+2 i a p$, which lies on the parabola $y^{2}=4 a^{2}\left(x+a^{2}\right)$, whose focus is at the origin and whose "positive axis" makes an angle zero with the initial line. (The positive axis of a parabola is the half-line starting at the vertex of the parabola and passing through its focus.) It follows that a line making an angle $\theta$ with the initial line is transformed by $\sigma$ to a parabola with focus at the origin, whose positive axis makes an angle $2 \theta$ with the initial line.

We embed the quasiregular tiling $(3,6,3,6)$ in the complex plane, using any point in the plane of the tiling as origin, and any direction for the initial line (see Fig. 12); denote this tiling in the complex plane by $\mathbf{H}$. Then $\sigma$ maps $\mathbf{H}$ to a tiling $\sigma \mathbf{H}$ of triangles and hexagons covering the two-sheeted surface, whose curvilinear edges meet at angles of

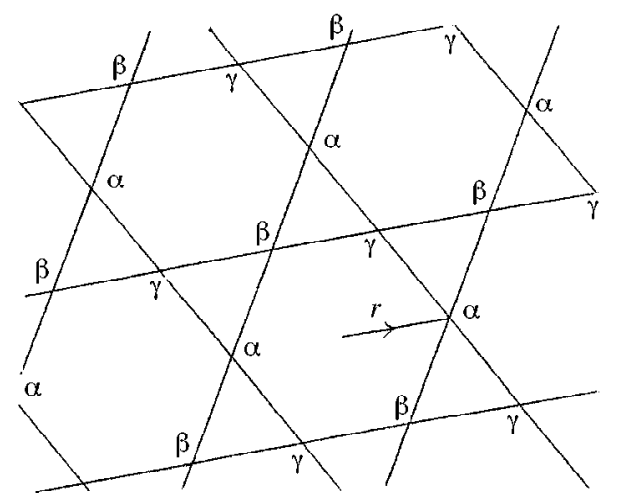

Fig. 12 


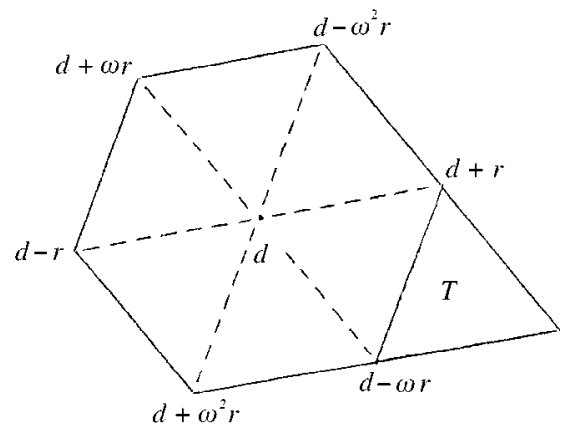

Fig. 13

$60^{\circ}$ and are formed by three families of parabolas with foci at the origin. The positive axes of the parabolas make angles of $120^{\circ}$ with each other. We aim to distort this tiling to obtain an h-tiling.

The tiling in Fig. 12 has three types of vertices, which we call $\alpha$-, $\beta$ - and $\gamma$-vertices as shown in the figure. All the hexagons are congruent, with their edges in the same three directions. Suppose that the vector from the centre of one hexagon to one of its $\alpha$-vertices is represented by the complex number $r$. Then all the hexagons have circumradius $|r|$, and $r$ represents the vector from the centre of any hexagon to one of its $\alpha$-vertices. We define the mapping $\tau$, which is confined to the vertices of the tiling and the centres of the hexagons, as follows:

If $d$ is the centre of any hexagon (i.e. if the centre of the hexagon is represented by the complex number $d$ ), then $\tau(d)=d^{2}$; if $a$ is an $\alpha$-vertex, then $\tau(a)=a^{2}-r^{2}$; if $b$ is a $\beta$-vertex, then $\tau(b)=b^{2}-\omega^{2} r^{2}$; if $c$ is a $\gamma$-vertex, then $\tau(c)=c^{2}-\omega r^{2}$, where $\omega=(-1+i \sqrt{3}) / 2$ is a complex cube root of 1 .

We can think of $\tau$ as a slight distortion of $\sigma$, confined to certain points only.

Consider a typical hexagon from Fig. 12, shown magnified in Fig. 13, with centre $d$. The $\alpha$-vertices of this hexagon are $d \pm r$, the $\beta$-vertices are $d \pm \omega r$, and the $\gamma$-vertices are $d \pm \omega^{2} r$. When this hexagon is transformed by $\tau$, the transformed centre is $\tau(d)=d^{2}$, the transformed $\alpha$-vertices are $\tau(d \pm r)=d^{2} \pm 2 d r$, the transformed $\beta$-vertices are $\tau(d \pm \omega r)=d^{2} \pm 2 \omega d r$, and the transformed $\gamma$-vertices are $\tau\left(d \pm \omega^{2} r\right)=d^{2} \pm 2 \omega^{2} d r$. Thus the transformed hexagon is regular, with centre $d^{2}$ and circumradius $|2 d r|$, and hence the vertices in Fig. 12, when transformed by $\tau$, form an h-tiling, in which the circumradius of any hexagon is proportional to the square root of the distance of its centre from the origin. Note that $\tau$ does not change the orientation of the hexagons.

Denote by $\tau \mathbf{H}$ the transform by $\tau$ of the vertices of $\mathbf{H}$, with two transformed vertices being joined by a line-segment whenever the original vertices were joined by an edge of $\mathbf{H}$. The vertices of $\sigma \mathbf{H}$ certainly lie on a two-sheeted surface with a branch point at the origin. Since the vertices of $\tau \mathbf{H}$ are obtained by distorting or moving the vertices of $\sigma \mathbf{H}$ through a fixed distance $\left|r^{2}\right|$, each vertex of $\tau \mathbf{H}$ lies on a well-defined sheet of the two-sheeted surface, except that if $V$ is a vertex of $\mathbf{H}$ whose distance $|V|$ from the origin is less than $r$, then $|\sigma V|<\left|r^{2}\right|$ and it is not clear on which sheet $\tau V$ lies. 
After we have shown (in Theorem 4.1 below) that every h-tiling can be obtained in the form $\tau \mathbf{H}$, by choosing a suitable origin and initial line in the plane of $(3,6,3,6)$, we can then say that every h-tiling closes up after wrapping itself twice around its focal point. However, it is still unclear exactly what happens near the focal point, even though the vertices and edges of the tiling are precisely determined by $\tau \mathbf{H}$.

In the previous paragraph we considered the vertices and edges of the tiling, but there is an extra requirement that can be used to give a stronger definition of a double tiling: a tiling is a double tiling if every point of the plane that is not on an edge of the tiling belongs to exactly two positive tiles or to $(n+2)$ positive tiles and $n$ negative tiles; we must include the last possibility because an h-tiling will include negative triangular tiles, as is shown in the example in Fig. 6. Although we have shown that the vertices of an h-tiling wrap themselves twice around the focal point, we only conjecture that every h-tiling satisfies the stronger requirement for being a double tiling, and all the triangular tiles of an h-tiling are positive triangles except for a few triangles near the focal point.

The centres of the hexagons in Fig. 12 lie on three families of parallel lines, parallel to the edges of the tiling and forming an equilateral triangular grid; so the centres of the $\tau$-transforms of the hexagons lie on three families of parabolas, all with their foci at the origin and with their positive axes making angles of $120^{\circ}$ with each other; the three parabolas meeting at each centre make angles of $60^{\circ}$ with each other. The $\alpha$-vertices in Fig. 12 lie on three families of parallel lines, again parallel to the edges of the tiling, so the $\tau$-transforms of the $\alpha$-vertices lie on three families of parabolas with their foci at $-r^{2}$, and with their positive axes in the same three directions as before. Similarly the $\tau$-transforms of the $\beta$ - and $\gamma$-vertices each lie on three families of parabolas with their foci at $-\omega^{2} r^{2}$ and $-\omega r^{2}$, respectively.

Theorem 4.1. Every h-tiling can be obtained by the method described above, using a $\tau$-transformation with the focal point of the tiling as origin.

Proof. Consider a triangle $A B C$ of an h-tiling. This triangle completely determines the tiling, so we need only show that $A B C$ can be obtained as the $\tau$-transform of a triangle of a $(3,6,3,6)$ tiling $\mathbf{H}$, using the left isodynamic point $\Omega_{2}$ of $A B C$ as origin. We therefore choose $\Omega_{2}$ as origin. To see how the vertices of $A B C$ can be expressed by complex numbers when $\Omega_{2}$ is the origin, we make use of Theorem 2.5 and Fig. 11. The triangle $F G H$ is a negative equilateral triangle, so we can represent its vertices by complex numbers as follows: $H=\bar{x}, G=\bar{x}+\bar{y}$ and $F=\bar{x}-\omega^{2} \bar{y}$. We may suppose that the circle of inversion used to invert $A, B, C$ to $F, G, H$ is the unit circle. Inversion in the unit circle transforms $\bar{z}$ to $z^{-1}$, and hence $A, B, C$ are the points $(x-\omega y)^{-1},(x+y)^{-1}$ and $x^{-1}$, which may be rewritten as $t^{2} x(x+y), t^{2} x(x-\omega y)$ and $t^{2}(x+y)(x-\omega y)$, where $t^{2}$ denotes the complex number $[x(x+y)(x-\omega y)]^{-1}$.

Consider now the triangle in Fig. 13 labelled $T$; its $\alpha$-, $\beta$ - and $\gamma$-vertices are $d+r$, $d-\omega r$ and $d+r-\omega r$. The $\tau$-transforms of these points are $d^{2}+2 d r, d^{2}-2 \omega d r$ and $d^{2}+2(1-\omega) d r-4 \omega r^{2}$. We easily check that these points coincide with $A, B$ and $C$ if $d=t x$ and $r=\frac{1}{2} t y$. Thus, with these values of $d$ and $r, A B C$ is the $\tau$-transform of a triangle of $\mathbf{H}$, and the origin is the left isodynamic point of $A B C$. This completes the proof. 
When we obtain a double tiling by applying the $\tau$-transformation to the quasiregular tiling $\mathbf{H}$ in Fig. 12, the two layers of the double tiling will coincide only if $\mathbf{H}$ has central symmetry about the origin. This will occur only if the origin is either at the centre of a hexagon or at a vertex of the tiling. The two cases give rise to the special tilings shown in Figs. 7 and 8. It is best to regard these not as simple tilings but as double tilings with two coincident layers. At every vertex in Fig. 8, apart from the focal point (indicated by a dot), there are two hexagons and two triangles; to obtain this situation at the focal point we must count the hexagon and the negative triangle twice. In Fig. 7 the three lines converging at the focal point must be regarded as degenerate triangles, each having two coincident vertices and angles of $90^{\circ}, 90^{\circ}$ and $0^{\circ}$. This provides us with three coincident vertices of the tiling at the focal point; to obtain a hexagon of zero area at the focal point surrounded by six degenerate triangles (rather than a triangle of zero area surrounded by three degenerate triangles) we need to use two layers of the tiling.

\section{References}

1. H. Fukagawa and D. Pedoe, Japanese Temple Geometry Problems, Charles Babbage Research Center, Winnipeg, 1989.

2. R. A. Johnson, Advanced Euclidean Geometry, Dover, New York, 1960.

3. C. Kimberling, Central points and central lines in the plane of a triangle, Mathematics Magazine 67 (1994), 163-187.

4. I. M. Yaglom, Complex Numbers in Geometry, Academic Press, New York, 1968.

Received February 12, 1999, and in revised form October 25, 1999. Online publication May 16, 2000. 